\title{
Involvement of a FAD-Linked Oxidase RSc0454 for Expression of the Type III Secretion System and Pathogenicity in Ralstonia solanacearum
}

\author{
Min Chen, ${ }^{1}$ Nan Chen, ${ }^{2}$ Jiwu Wang, ${ }^{1}$ YuJian Zhou, ${ }^{3}$ Liangliang Han, ${ }^{1}$ Xiaojun Shi, ${ }^{1,4}$ Yasufumi Hikichi, ${ }^{5}$ \\ Kouhei Ohnishi, ${ }^{5}$ Jing $\mathrm{Li}^{6, \dagger}$ and Yong Zhang ${ }^{1,4, \dagger}$ \\ ${ }^{1}$ College of Resources and Environment, Southwest University, Chongqing, China \\ ${ }^{2}$ College of Chemistry and Chemical Engineering, Chongqing University of Science and Technology, Chongqing, China \\ ${ }^{3}$ Laboratory of Human Micromorphology, Chongqing Medical University, Chongqing, China \\ ${ }^{4}$ Interdisciplinary Research Center for Agriculture Green Development in Yangtze River Basin, Southwest University, \\ Chongqing, China \\ ${ }^{5}$ China Faculty of Agriculture and Marine Science, Kochi University, Nankoku, Japan \\ ${ }^{6}$ The Ninth Peoples Hospital of Chongqing, Chongqing, China
}

Accepted 10 August 2021.

\begin{abstract}
Ralstonia solanacearum RSc0454 is predicted as a FADlinked oxidase based on protein homologies, while it contains distinct domains of lactate dehydrogenase and succinate dehydrogenase. A previous study demonstrated that RSc0454 exhibits lactate dehydrogenase activity using pyruvate and NADH as substrates, and is essential for pathogenicity of $R$. solanacearum. Here, we genetically characterized involvement of RSc0454 on bacterial growth and expression of genes for the type III secretion system (T3SS, a pathogenicity determinant) in $R$. solanacearum. The $R S c 0454$ mutant grew normally in rich medium but grew faintly in host plants, and failed to grow in minimal medium. Supplementary succinate but not lactate could substantially restore some phenotypes of RSc0454 mutants, including faint growth in host plants, diminished growth in the minimal medium, and lost pathogenicity toward host plants. Expression of T3SS genes is directly controlled by a master regulator, $\mathrm{HrpB}$, and $h r p B$ expression is positively regulated by HrpG and PrhG in parallel ways. Deletion of $\mathrm{RSc0454}$ substantially reduced expression levels of $h r p B$ and T3SS both in vitro and in planta. Moreover, RSc0454 is revealed to be required for the T3SS expression via HrpG and PrhG, although through some novel pathway, and impaired expression of these genes was not due to growth deficiency of $\mathrm{RSc0454}$ mutants. RSc0454 is suggested to be important for redox balance inside cells, and
\end{abstract}

${ }^{\dagger}$ Corresponding authors: Y. Zhang; bioyongzhang@swu.edu.cn, and J. Li; hellolijing@126.com

M. Chen and N. Chen contributed equally to this work.

Funding: Support was provided by the Chongqing Research Program of Basic Research and Frontier Technology grant cstc2015jcyjA10011 and the National Natural Science Foundation of China grant 31670082.

*The $\boldsymbol{e}$-Xtra $\log$ stands for "electronic extra" and indicates that a supplementary table and supplementary figures are published online.

The author(s) declare no conflict of interest.

(c) (1) () $\odot$ Copyright $\odot 2021$ The Author(s). This is an open access article distributed under the CC BY-NC-ND 4.0 International license. supplementary NADH partially restored diminished growth of the RSc0454 mutant in the minimal medium only in the presence of succinate at some moderate concentrations, indicating that the unbalanced redox in the $R S c 0454$ mutant might be responsible for its diminished growth in the minimal medium. Taken together, these results provide novel insights into the understanding of various biological functions of this FAD-linked oxidase RSc0454 and involvement of the redox balance on expression of the T3SS in $R$. solanacearum.

Keywords: bacterial pathogenesis, bacteria-plant symbiosis, genetics and gene regulation, type- 3 secretion

Ralstonia solanacearum is a causal agent of bacterial wilt diseases on a wide variety of plant species and currently is regarded as one of the most destructive plant-pathogenic bacteria worldwide due to its ability to cause severe yield losses in many economically important crops (Genin 2010; Mansfield et al. 2012). $R$. solanacearum is a soilborne, Gram-negative, vascular bacterium that generally invades host xylem vessels through root wounds or natural openings (Vasse et al. 1995). Once bacteria have invaded xylem vessels, they proliferate extensively and produce a huge amount of exopolysaccharide (EPS) slime, which blocks sap flows in xylem vessels, resulting in quick stunting and wilting of host plants and is believed to be one of the main virulence factors (Denny 1995; Roberts et al. 1988). In addition to the EPS, a syringe-like type III secretion system (T3SS) is another pathogenicity determinant of $R$. solanacearum that plays an essential role in the infection process of $R$. solanacearum, especially at early stages, to invade host cells (Angot et al. 2006; Cunnac et al. 2004; Jones and Dangl 2006). The T3SS is conserved in many pathogenic bacteria of animals and plants, and bacteria use this syringe-like system to inject virulence factors (so-called type III effectors [T3Es]) into host cytosol to subvert host defense (Jones and Dangl 2006; Poueymiro and Genin 2009).

The $R$. solanacearum T3SS is encoded by 22 genes forming a hypersensitive response and pathogenicity ( $h r p$ ) gene cluster (Arlat et al. 1992), and is globally regulated by a complex network (Genin and Denny 2012; Hikichi et al. 2017). In general, an $\mathrm{AraC}$ family of transcriptional regulator $\mathrm{HrpB}$ directly controls 
expression of genes for the T3SS and T3Es (Coll and Valls 2013; Mukaihara et al. 2010). Genes of $h r p B$, T3SS, and T3Es are not expressed in a nutrient-rich medium but are activated in a nutrient-limited medium (the minimal medium) that might mimic plant apoplastic fluids, or conditions getting in contact with host signals (Marenda et al. 1998; Yoshimochi et al. 2009b; Zhang et al. 2013). Two close paralogs, HrpG and PrhG, OmpR/PhoB family of response regulators of the two-component systems, can respond to host signals, or some mimic signals by phosphorylation and positively regulate $h r p B$ expression in parallel ways (Plener et al. 2010; Zhang et al. 2013). Although HrpG is well expressed in nutrient-rich medium, its regulation on expression of $h r p B$ and the T3SS genes is only activated by host signals or mimic signals (i.e., in the minimal medium). Host signals are presumed to be recognized by PrhA, an outer membrane protein, and transferred to HrpG through the PrhA-PrhR/I-PrhJ signaling cascade or some novel signaling cascades (Aldon et al. 2000; Marenda et al. 1998; Valls et al. 2006; Yoshimochi et al. 2009b). Moreover, a transcriptional regulator of the LysR-family, PhcA, negatively regulates expression of the T3SS, which represses $\mathrm{prhI} / \mathrm{R}$ expression at high cell density by binding to $\mathrm{prhI} / \mathrm{R}$ promoter and, in turn, shuts down expression of prhJ, hrpG, hrpB, and the T3SS (Genin et al. 2005; Yoshimochi et al. 2009a). The prh $G$ expression is independent from the PrhA signaling cascade and positively regulated by $\mathrm{PhcA}$, PrhN, and some other regulators (Zhang et al. 2013, 2015).

To further elucidate complex regulation of the $R$. solanacearum T3SS, we previously screened a subset of T3SS-regulating candidates with transposon mutagenesis (Zhang et al. 2013). Among them is $\mathrm{RSc} 0454$ (1,345 amino acids), which is predicted to be a FAD-linked oxidase based on protein homologies, while it contains distinct domains of N-terminal DUF3683, lactate dehydrogenase (LDH), and succinate dehydrogenase (SDH). A pioneering study demonstrated that $R$. solanacearum RSc0454 shows catalytic activities of both LDH and SDH using pyruvate and NADH as substrates, and is essential for pathogenicity of $R$. solanacearum toward tomato plants ( $\mathrm{Hu}$ et al. 2019). It remains unclear why the RSc0454 mutant fails to cause wilt disease on host plants. The T3SS expression was substantially reduced in $\mathrm{RSc} 0454$ transposon mutants. It is the first report linking the T3SS with FAD-linked oxidase. Thus, we focused on RSc0454 to investigate its functional contribution to the T3SS expression and pathogenicity in $R$. solanacearum.

The FAD-linked oxidases are known to catalyze vital redox reactions, especially for basic metabolism in many microbes (Gao et al. 2015; Heikal et al. 2014). We first evaluated growth profiles of $R S c 0454$ mutants. Deletion of $R S c 0454$ does not alter growth of $R$. solanacearum in a rich medium (Hu et al. 2019). Here, we shifted to evaluate its growth in a minimal medium (Hoagland medium supplemented with $2 \%$ of sucrose), which is used to induce the T3SS expression in some studies. RSc0454 of GMI1000 and OE1-1 are identical in amino acids (data not shown). We generated an RSc0454 mutant (RQ5699) from OE1-1 with methods for the in-frame deletion of target genes (Zhang et al. 2015). The $R$. solanacearum wild-type strain and its derivative mutants used in this study are listed in Table 1. RQ5699 exhibited a growth profile similar to that of parent strain (RK5050), a popA-lacZYA reporter strain of OE1-1 in the nutrient-rich medium (broth medium), while it failed to grow in the minimal medium (Fig. 1A). The genetic complementation assay was performed with the Tn7-based site specific chromosomal integration system (Choi et al. 2005; Zhang et al. 2021), and the complementation strain (RQC307) fully restored its diminished growth in the minimal medium (Fig. 1A and B), confirming that RSc0454 is essential for $R$. solanacearum to grow in the minimal medium.

Supplementary succinate and lactate restore some phenotypes of the GMI1000 RSc0454 mutant (i.e., cell aggregation at the bottom of the rich medium with stationary culture) ( $\mathrm{Hu}$ et al. 2019). We assessed whether supplementary succinate or lactate could restore diminished growth of RQ5699 in the minimal medium. Growth of RQ5699 was substantially restored only with $40 \mathrm{mM}$ succinate, whereas growth of the wild-type strain was significantly repressed in this medium (Fig. 1C). The addition of $100 \mathrm{mM}$ succinate resulted in no growth of either the wild-type strain or the $R S c 0454$ mutant. Note that supplementary lactate at concentrations of $5 \mathrm{mM}$ or higher completely diminished growth of both the wild-type strain and RSc0454 mutant in the minimal medium (data not shown), while that at $1 \mathrm{mM}$ did not affect growth of the wild-type strain in the minimal medium. Therefore, lactate was added into the minimal medium at concentrations of less than $1 \mathrm{mM}$ for the growth assay. Supplementary lactate at any concentration did not restore cell grow of the RSc0454 mutant in the minimal medium (Fig. 1D; Supplementary Fig. S1). All of these results indicate that high concentration of succinate is generally toxic to $R$. solanacearum cells and RSc0454 could be used to supply succinate from fumarate by reverse reaction of $\mathrm{SDH}$.

$\mathrm{RSc} 0454$ is essential for pathogenicity of $R$. solanacearum GMI1000 on tomato plants (Hu et al. 2019). GMI1000 wilts tomato plants but elicits hypersensitive response in tobacco leaves, while OE1-1 is virulent in tomato and tobacco plants (Kanda et al. 2003; Poueymiro et al. 2009; Salanoubat et al. 2002). We assessed whether RSc0454 is required for OE1-1 to wilt tomato and tobacco plants. Tomato plants were challenged with the inoculation method of petiole inoculation, which drops $2 \mu \mathrm{l}$ of bacterial suspension at a density of $10^{8} \mathrm{CFU} \mathrm{ml^{-1 }}$ onto the fresh-cut surface of tomato petioles, and tobacco plants were challenged with the inoculation method of leaf infiltration, which infiltrates approximately $50 \mu \mathrm{l}$ of bacterial suspension at a density of $10^{8} \mathrm{CFU} \mathrm{ml} \mathrm{m}^{-1}$ into tobacco leaves with a needleless syringe (Zhang et al. 2015). RQ5699 failed to cause disease on tomato and tobacco plants, and complementation strain RQC307 fully restored its diminished pathogenicity to that of the parent strain on tomato and tobacco plants (Fig. 2A and B), confirming that the essential role of $\mathrm{RSc} 0454$ on pathogenicity is conserved in different $R$. solanacearum strains and different host plants. Supplementary succinate significantly restored growth of the RSc0454 mutant in the minimal medium (Fig. 1C). We assessed whether supplementary succinate could restore diminished pathogenicity of RQ5699 on host plants. Approximately 2- to 3week-old tomato and tobacco plants were irrigated with onequarter-diluted Hoagland medium with supplementary succinate at a final concentration of $40 \mathrm{mM}$ for 7 days and subjected to the virulence assay. Supplementary succinate did not alter virulence of the wild-type strain on tobacco and tomato plants, whereas it somehow enabled RQ5699 to wilt tobacco and tomato plants (Fig. 2A and B), indicating that diminished pathogenicity of the $\mathrm{RSc} 0454$ mutant is possibly due to insufficient succinate in host plants.

The in planta proliferation is one of the most important pathogenicity determinants of $R$. solanacearum (Genin and Denny 2012). We assessed whether $R S c 0454$ deletion impaired $R$. solanacearu proliferation in host plants. For the growth assay in tobacco leaves, a bacterial suspension at a density of $10^{4} \mathrm{CFU}$ $\mathrm{ml}^{-1}$ was infiltrated into tobacco leaves and cell discs were punched for quantification of cell densities every other day (Zhang et al. 2015). The wild-type strain RK5050 proliferated extensively in tobacco leaves that reached the maximum of $10^{9}$

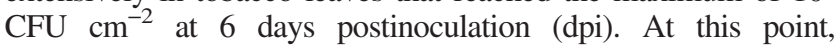
RK5050-infiltrated leave became withered and dried. Proliferation of RQ5699 in tobacco leaves that reached the maximum of approximately $10^{5} \mathrm{CFU} \mathrm{\textrm {cm } ^ { - 2 }}$ at $4 \mathrm{dpi}$ was substantially impaired, and the leaves did not grow any more (Fig. 3A). For the growth assay in tomato stems, tomato plants were inoculated 
by dropping $2 \mu \mathrm{l}$ of bacterial suspension at a density of $10^{8}$ $\mathrm{CFU} \mathrm{ml} \mathrm{m}^{-1}$ onto fresh-cut surfaces of petioles, and stem pieces were cut for quantification of cells densities from $3 \mathrm{dpi}$. RK5050 proliferated extensively in tomato stems that reached the maximum of approximately $10^{10} \mathrm{CFU} \mathrm{g}^{-1}$ at 5 to $6 \mathrm{dpi}$ (Fig. 3B). At this point, RK5050-inoculated tomato plants became withered and dried. Proliferation of RQ5699 in tomato stems that reached the maximum of approximately $10^{4} \mathrm{CFU} \mathrm{g}^{-1}$ at 3 to 4 dpi was significantly impaired, and the stems did not grow any more (Fig. 3B). Supplementary succinate significantly restored growth of the $\mathrm{RSc} 0454$ mutant in the minimal medium. We further assessed whether supplementary succinate could restore proliferation of the $R S c 0454$ mutant in host plants. Tomato and tobacco plants were irrigated with one-quarterdiluted Hoagland medium supplemented with succinate at a final concentration of $40 \mathrm{mM}$ and the in planta growth assay was performed. Proliferation of RQ5699 in succinate-irrigated tobacco leaves was significantly increased and reached the maximum of approximately $10^{8}$ to $10^{9} \mathrm{CFU} \mathrm{cm^{-2 }}$ at 9 dpi (Fig. 3A). At this point, RQ5699-infiltrated tobacco leaves became withered and dried. In succinate-irrigated tomato stems, RQ5699 proliferated to the maximum of approximately $10^{9} \mathrm{CFU} \mathrm{g}^{-1}$ at 6 to $9 \mathrm{dpi}$ (Fig. 3B). All of these results indicate that RSc0454 is important for the in planta proliferation of $R$. solanacearum, and impaired in planta proliferation of the $R S C 0454$ mutant is possibly due to insufficient succinate in host plants.

The RSc0454 mutant was originally screened as one of the T3SS-regulating candidates, in which popA expression was substantially reduced (Zhang et al. 2013). In our study, expression profiles of the T3SS genes were monitored by a popA-lacZYA fusion, which does not alter the infection process of OE1-1 on host plants (Zhang et al. 2013). The T3SS genes are not expressed in rich medium but are induced in the minimal medium (called hrp-inducing medium) (Yoshimochi et al. $2009 b$ ). We first assessed whether RSc0454 is required for popA expression in $R$ solanacearum. Because $\mathrm{RSc} 0454$ mutants failed to grow in the minimal medium, we added $40 \mathrm{mM}$ succinate to support cell growth in this medium. The popA expression was much lower in the RSc0454 mutant (RQ5699) and the complementation strain RQC307 fully restored popA expression to the level of the wild type (Fig. 4A). The T3SS expression can be greatly enhanced in planta or under conditions getting in contact with plant signals compared with that in the minimal medium (Valls et al. 2006; Yoshimochi et al. 2009b). We assessed whether RSc0454 is required for popA expression in host plants. The in planta enzyme assay was performed in tobacco leaves, in which a bacterial suspension at $10^{8} \mathrm{CFU} \mathrm{ml}^{-1}$ was infiltrated into tobacco leaves and leaf discs were punched for the in planta
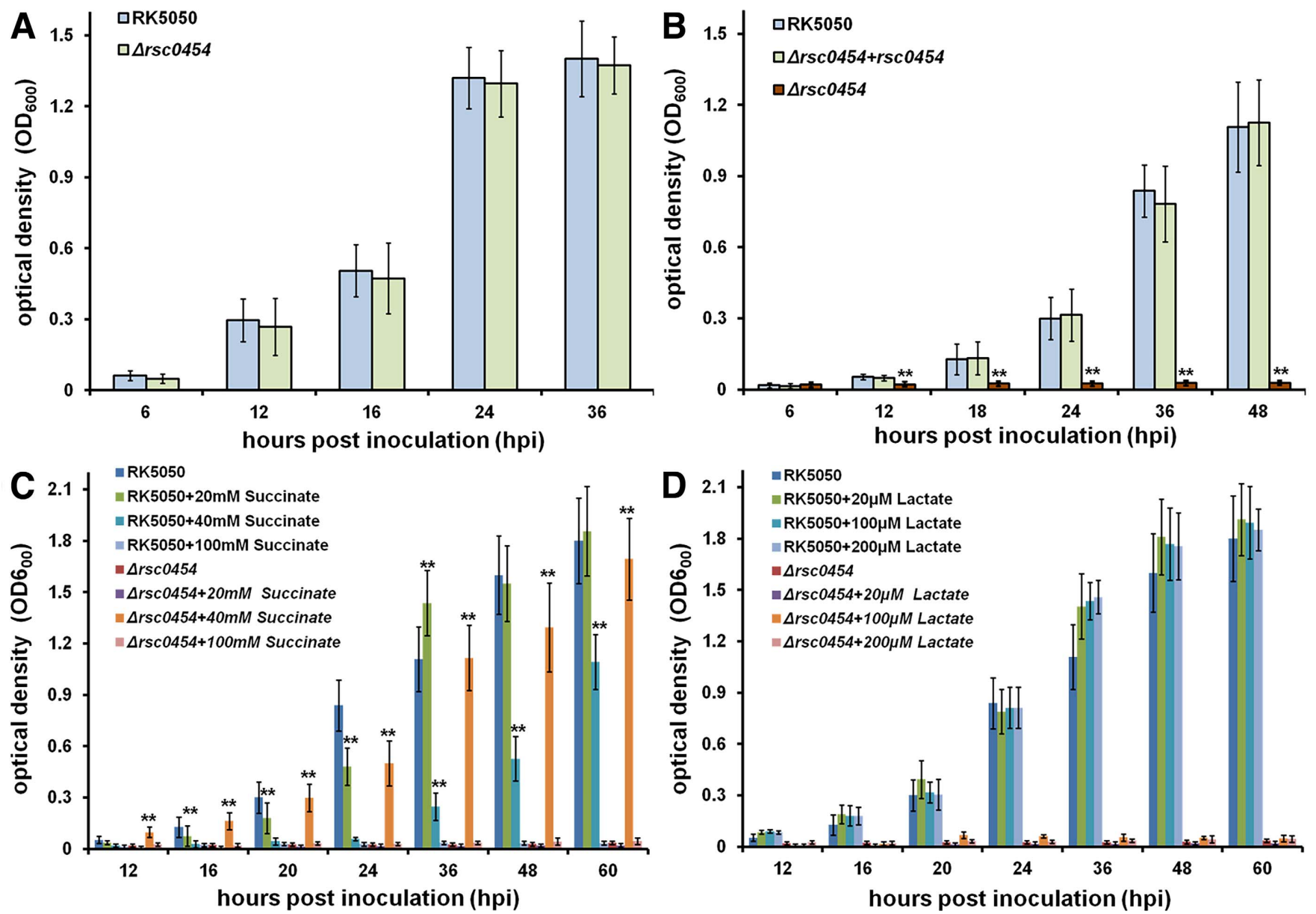

Fig. 1. Involvement of RSc0454 on growth of Ralstonia solanacearum in A, nutrient-rich broth medium; $\mathbf{B}$, nutrient-limited minimal medium; and $\mathbf{C}$ and $\mathbf{D}$, minimal medium supplemented with succinate and L-lactate at different concentrations. RK5050 $(\mathrm{OE} 1-1$, popA-lacZYA $)=$ the wild-type strain, $\triangle R S c 0454=$ $\mathrm{RQ} 5699(\mathrm{RK} 5050=\Delta R S c 0454)$, and $\Delta R S c 0454+R S c 0454=\mathrm{RQC0307}$ (complementary $R S c 0454$ in RQ5699). For the growth assay in medium, a cell suspension was washed twice with distilled water, adjusted to an optical density at $600 \mathrm{~nm}\left(\mathrm{OD}_{600}\right)$ of 1.0 , inoculated into fresh broth medium and minimal medium (Hoagland medium supplemented with $2 \%$ of sucrose) with a proportion of $1 \%$, and $\mathrm{OD}_{600}$ was measured periodically. Mean values of all three biological replicates with three replicates per trial were averaged with standard deviation (error bars). Statistical significance between $R S c 0454$ mutants and RK5050 was assessed using a post hoc Dunnett test following analysis of variance. Significance level $* *$ indicates $P<0.01$. 
enzyme assay at 6 to $24 \mathrm{~h}$ postinoculation (hpi) (Zhang et al. 2011). The popA expression in RQ5699 was significantly impaired in tobacco leaves, and complementary RSc0454 fully restored its impaired popA expression to that of the wild-type strain in tobacco leaves (Fig. 4B). On the other hand, the popA expression in RQ5699 and RK5050 was not improved even when succinate was supplied for irrigation of tobacco plants
(Fig. 4B), confirming that RSc0454 is important for the T3SS expression both in vitro and in planta, and impaired T3SS expression in the $\mathrm{RSc0454}$ mutant is not because of growth deficiency.

T3SS genes are directly controlled by HrpB and $h r p B$ expression is positively regulated by $\mathrm{HrpG}$ and PrhG, which are regulated by PrhJ, PhcA, and PrhN, respectively. Involvement of
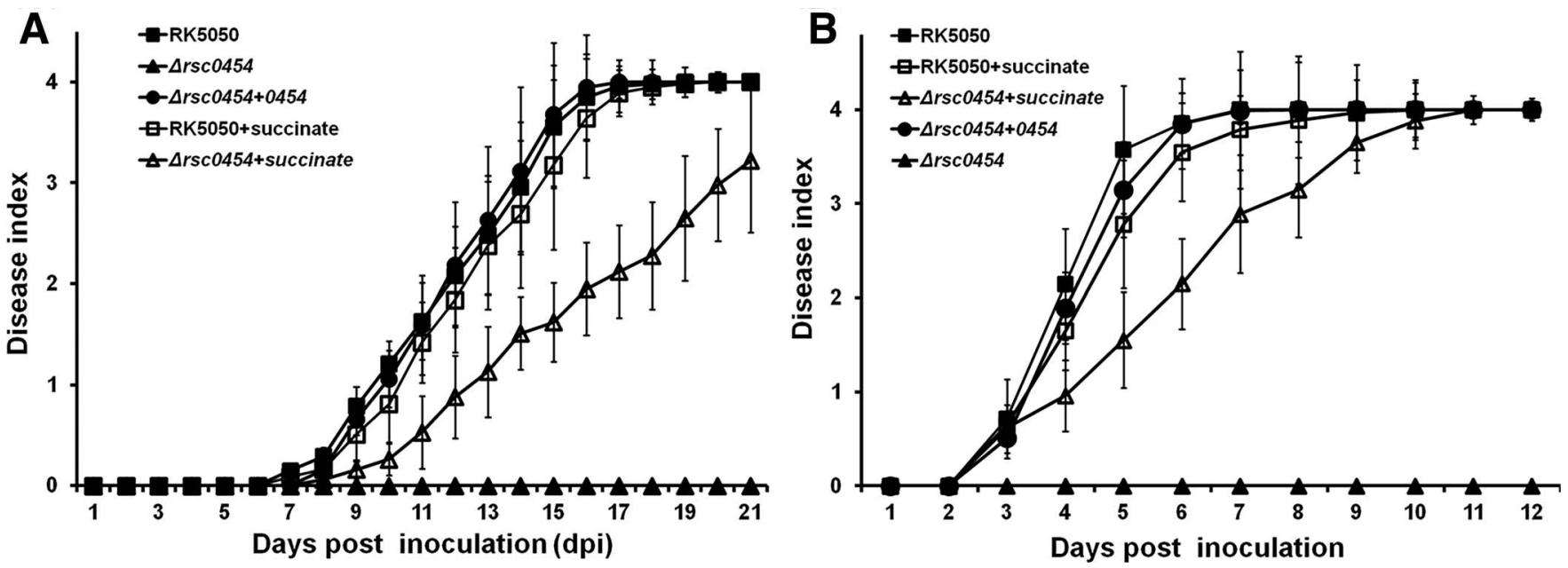

Fig. 2. Virulence assay of the $R S c 0454$ mutant on $\mathbf{A}$, tomato plants with the petiole inoculation method and $\mathbf{B}$, tobacco plants with the leaf infiltration inoculation method. RK5050 = the wild-type strain, $\Delta R S c 0454=$ RQ5699 $($ RK5050 = $\Delta R S c 0454), \Delta R S c 0454+R S c 0454=$ RQC0307 (complementary $R S c 0454$ in RQ5699), and $\Delta R S c 0454+$ succinate $=$ succinate-irrigated plants were subjected to the virulence assay. For the petiole inoculation, $2 \mu 1$ of bacterial suspension at $10^{8} \mathrm{CFU} \mathrm{ml}^{-1}$ was dropped onto the freshly cut surface of tomato petioles. For the leaf infiltration, approximately $50 \mu \mathrm{l}$ of bacterial suspension at $10^{8} \mathrm{CFU} \mathrm{ml}^{-1}$ was infiltrated into tobacco leaves with a blunt-end syringe. For succinate supplementation, approximately 2- to 3 -week-old plants were irrigated with one-quarter-diluted Hoagland medium containing succinate $(40 \mathrm{mM})$ for 7 days and subjected to a virulence test. Wilt symptoms were inspected daily and scored on a disease index scale from 0 to 4 , where $0=$ no wilting, $1=1$ to $25 \%$ wilting, $2=26$ to $50 \%$ wilting, $3=51$ to $75 \%$ wilting, and $4=76$ to $100 \%$ wilted or dead. Each assay was repeated with at least four biological replicates including 12 plants per trial. Mean values of all experiments were averaged with standard deviation (error bars).
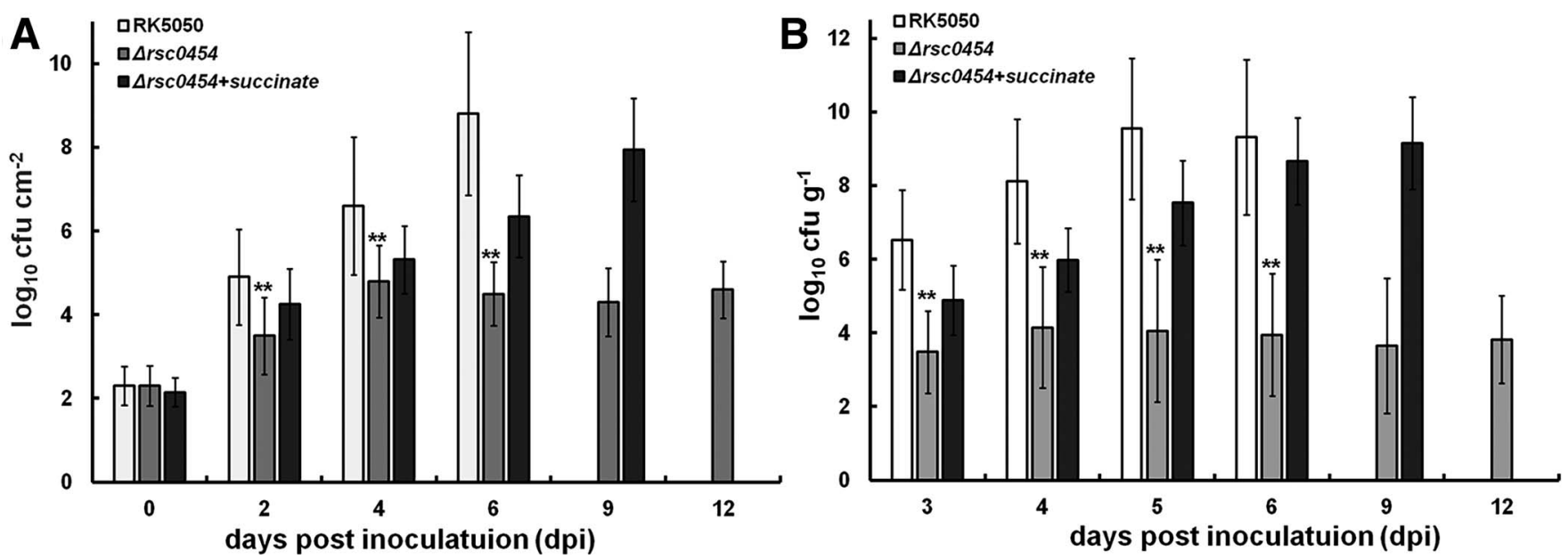

Fig. 3. Involvement of RSc0454 on growth of Ralstonia solanacearum in A, tobacco leaves (intercellular spaces) and $\mathbf{B}$, tomato stems (xylem vessels). RK5050 (OE1-1, popA-lacZYA), $\Delta R S c 0454=$ RQ5699 $(\mathrm{RK5050}=\Delta R S c 0454)$, and $\Delta R S c 0454+$ succinate $=$ succinate-irrigated plants were subjected to the growth assay. For the growth assay in tobacco leaves, which was presented in log CFU per square centimeter, tobacco plants were inoculated with the leaf infiltration method and leaf disks $\left(0.38 \mathrm{~cm}^{2}\right)$ were punched and subjected to quantification of cells number by dilution plating every other day. The RK5050-inoculated tobacco leaves were punched till 6 days postinoculation (dpi), when tobacco leaves became withered and dried, while the RQ5699-inoculated tobacco leaves were assessed till $12 \mathrm{dpi}$, when tobacco leaves remained healthy but became yellow. For the growth assay in tomato stems, which was presented in log CFU per gram, tomato plants were inoculated with the petiole inoculation method and stem species were cut, weighed, and subjected to quantification of cells number by dilution plating on a daily basis from 3 dpi. The RK5050-inoculated tomato stems were harvested till 6 dpi, when tomato plants died, while the RQ5699-inoculated tomato stems were harvested till 12 dpi. For the succinate supplementation, approximately 2- to 3-week-old tomato and tobacco plants were irrigated with one-quarter-diluted Hoagland medium containing succinate for 7 days and subjected to the in planta growth assay. Each assay was repeated with at least four biological replicates including six replicates per trial. Mean values from all experiments were averaged and presented with standard deviation (error bars). Statistical significance between RQ5699 and RK5050, or that between treatment with supplementary succinate or without, was assessed using a post hoc Dunnett test following analysis of variance. Significance level ** indicates $P<0.01$. 
RSc0454 in expression of these genes was assessed. Expression of $h r p B$ and the T3SS was not expressed in the nutrient-rich medium but was activated in the minimal medium. Expressions of $h r p B, h r p G$, and $p r h G$ were reduced in $R S c 0454$ mutants in the minimal medium and addition of succinate for cell growth did not alter expression of these genes in the minimal medium (Fig. 4C). Expression of $h r p G$ and $p r h G$ in $R S c 0454$ mutants were very low even in the nutrient-rich medium, in which RSc0454 mutants grew normally (Supplementary Fig. S2). The complementary $\mathrm{RSc} 0454$ fully restored impaired expression of $h r p G$ and $p r h G$ to those of parent strains in nutrient-rich medium (data not shown). On the other hand, three genes (prhJ, $p h c A$, and $p r h N$ ) which regulate the T3SS expression indirectly expressed normally in RSc0454 mutants both in the minimal and rich media (Fig. 4C; Supplementary Fig. S2). Impact of $\mathrm{RSc0454}$ on $h r p B$ expression was also assessed in tobacco leaves. Deletion of $\mathrm{RSc} 0454$ significantly reduced $h r p B$ expression in tobacco leaves and supplementary succinate for irrigation of tobacco plants did not alter $h r p B$ expression in tobacco leaves (Fig. 4D). All of these results suggested that expressions of both $h r p G$ and $p r h G$ were reduced with $R S c 0454$ deletion regardless of growth conditions and it, in turn, impaired expression of the T3SS in RSc0454 mutants, and impaired expression of T3SS genes was not due to growth deficiency of $\mathrm{RSc} 0454$ mutants.

$\mathrm{RSc0454}$ is suggested to be involved in redox balance inside cells that is in line with different living conditions of $R$. solanacearum (Hu et al. 2019). We assessed whether supplementary $\mathrm{NADH}$ or $\mathrm{NAD}^{+}$could restore unbalanced redox in the RSc0454 mutant. Supplementary NADH at concentrations of 0.1 to $5 \mathrm{mM}$ or $\mathrm{NAD}^{+}$at $0.1 \mathrm{mM}$ did not alter growth of the wildtype strain, whereas supplementary $\mathrm{NAD}^{+}$at $5 \mathrm{mM}$ diminished growth of the wild-type strain in minimal medium (Supplementary Fig. S3A), indicating that excess $\mathrm{NAD}^{+}$might disturb redox status inside cells. Supplementary succinate at $40 \mathrm{mM}$ substantially restored diminished growth of the RSc0454 mutant in the minimal medium (Fig. 1C), indicating that unbalanced redox in
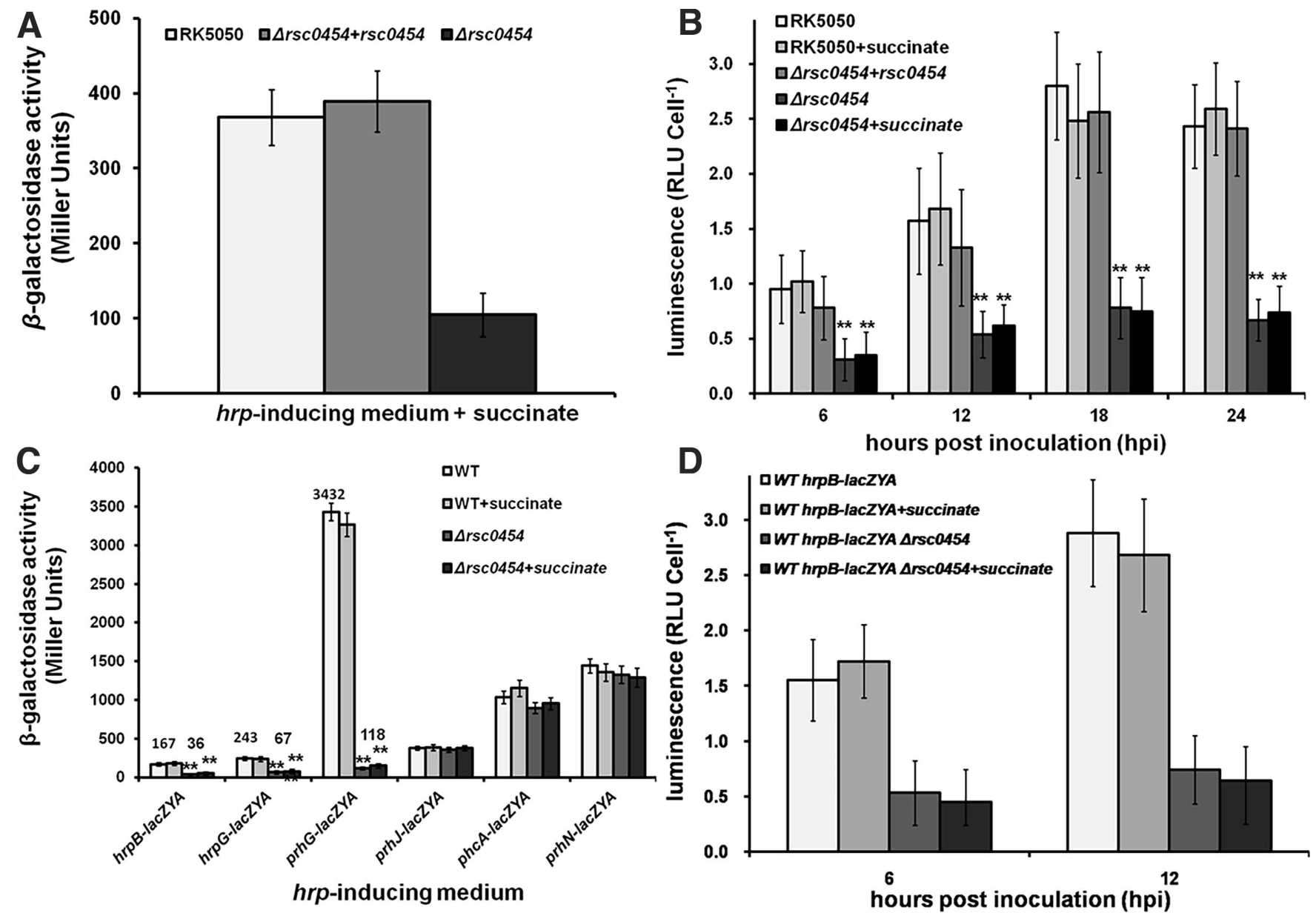

Fig. 4. Involvement of RSc0454 on expression of the type III secretion system (T3SS) and T3SS-regulated genes popA-lacZYA in A, hypersensitive response and pathogenicity ( $h r p$ )-inducing medium and B, tobacco leaves; C, expression of T3SS-regulated genes in $h r p$-inducing medium; and $\mathbf{D}$, expression of hrpB-lacZYA in tobacco leaves. WT = wild type. RK5050 (OE1-1, popA-lacZYA); $\triangle R S c 0454=$ deleted RSc0454 from reporter strains of hrpB-lacZYA, hrpG-lacZYA, prhG-lacZYA, prhJ-lacZYA, phcA-lacZYA, and prhN-lacZYA; $\triangle R S c 0454$ + 0454 (complementary RSc0454 in RQ5699); and $h r p$-inducing medium + succinate $=$ supplemented succinate into the minimal medium ( $h r p$-inducing medium) at a final concentration of $40 \mathrm{mM}$ that were subjected to cell growth. For the in vitro enzyme assay, strains were grown in medium to an optical density at $600 \mathrm{~nm}\left(\mathrm{OD}_{600}\right)$ of approximately 0.1 and subjected to the enzyme assay, enzymatic activities of which were presented in Miller Units. For the in planta enzyme assay, tobacco leaves were infiltrated with bacterial suspension at $\mathrm{OD}_{600}$ of 0.1 and leaf disks were punched for the enzyme assay with the Galacto-Light Plus kit, enzymatic activities of which were presented with luminescence normalized by cells number (relative luminescence units [RLU] per cell). For the in planta enzyme assay with supplementary succinate, succinate-irrigated tobacco leaves were used for cell infiltration. The in planta enzyme assay was carried out from 6 days postinoculation to $24 \mathrm{~h}$ postinoculation (hpi), when infiltrated leaves became withered and dried. Cells densities were quantified by dilution plating and luminescence was determined using the GloMax20 luminometer (Promega Corp.). Each assay was repeated with four biological replicates including six replications per trial. Mean values of all experiments were averaged with standard deviation and statistical significance between RK5050 and mutants was assessed using a post hoc Dunnett test following analysis of variance. Significance level ** indicates $P<0.01$. 
the $\mathrm{RSc} 0454$ mutant was somehow restored to some extent with supplementary succinate at this concentration. It is striking that supplementary $\mathrm{NADH}$ at $5 \mathrm{mM}$ began to diminish growth of the $R S c 0454$ mutant in the presence of succinate at $40 \mathrm{mM}$ in the minimal medium (Supplementary Fig. S3C). Supplementary $\mathrm{NADH}$ or $\mathrm{NAD}^{+}$at $0.1 \mathrm{mM}$ did not restore growth of the RSc0454 mutant in the minimal medium, whereas they began to partially restore growth of $\mathrm{RSc} 0454$ mutants only in the presence of succinate at $10 \mathrm{mM}$ in the minimal medium at 36 hpi (Supplementary Fig. S3B). Note that supplementary succinate at 10 $\mathrm{mM}$ did not restore growth of the $\mathrm{RSc} 0454$ mutant in the minimal medium. This was consistent with the fact that NADH and $\mathrm{NAD}^{+}$work as redox cofactors in reactions. Supplementary succinate and $\mathrm{NADH}$ and $\mathrm{NAD}^{+}$together restored redox balance inside $\mathrm{RSc0454}$ mutant cells. The unbalanced redox in the

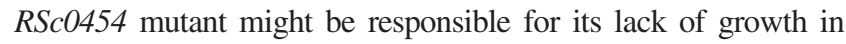
the minimal medium.

In summary, we provided multiple lines of evidence to demonstrate that $\mathrm{RSc0454}$ is important for $R$. solanacearum to grow under nutrient-limited conditions and required for expression of the T3SS genes both in vitro and in planta. The RSc0454 mutant is easily aggregated at the bottom of nutrient-rich medium with stationary culture, which is known to be insufficient in oxygen, indicating that the oxidase encoded by $\mathrm{RSc} 0454$ is possibly important for a redox balance inside cells (Hu et al. 2019). Succinate and fumarate are important components in the citric acid cycle and play important roles in the respiratory electron transfer chain in many microbes (Huynen et al. 1999; Park et al. 1999). Succinate and fumarate might restore the redox balance to some extent and, hence, can restore some phenotypes of the $\mathrm{RSc0454}$ mutant (i.e., cell aggregation) (Hu et al. 2019). Supplementary succinate at a higher concentration significantly impaired growth of the wild-type strain, especially at early stages of growth. It possibly perturbs the redox balance inside cells and, hence, is toxic for $R$. solanacearum. Supplementary succinate at moderate concentrations (i.e., $40 \mathrm{mM}$ ) substantially restores phenotypes of the RSc0454 mutant, including the faint growth in host plants, diminished growth in the minimal medium, and lost pathogenicity toward host plants. It might restore the redox balance inside
RSc0454 mutant cells to some extent. Excess NADH or NAD ${ }^{+}$ might disturb redox status inside cells, and supplementary $\mathrm{NADH}$ or $\mathrm{NAD}^{+}$at higher concentration (i.e., $5 \mathrm{mM}$ ) diminished growth of $R$. solanacearum. Supplementary succinate at $40 \mathrm{mM}$ might somehow restore redox balance inside the RSc0454 mutant because it significantly restores diminished growth of the $\mathrm{RSc0454}$ mutant in the minimal medium. It was consistent with the fact that supplementary $\mathrm{NAD}^{+}$at $5 \mathrm{mM}$ began to diminish growth of the $\mathrm{RSc0454}$ mutant in the minimal medium in the presence of succinate at $40 \mathrm{mM}$. Supplementary succinate at $10 \mathrm{mM}$ failed to restore growth of the $\mathrm{RSc} 0454$ mutant in the minimal medium, whereas supplementary NADH or NAD+ at $0.1 \mathrm{mM}$ began to restore growth of the $\mathrm{RSc} 0454$ mutant in the minimal medium in the presence of succinate at $10 \mathrm{mM}$, indicating that supplementary $\mathrm{NADH}$ or $\mathrm{NAD}^{+}$works as a redox cofactor to restore the redox balance inside $R S c 0454$ mutant cells in the presence of succinate at $10 \mathrm{mM}$. The unbalanced redox in the $\mathrm{RSc} 0454$ mutant might be responsible for its lack of growth in the minimal medium.

Nutrients in xylem sap are believed to be relatively poorer than that in nutrient-rich medium (i.e., broth medium) (Evert and Eichhorn 2006; Lowe-Power et al. 2018a; Pegg 1985). $R$. solanacearum could utilize some compounds in xylems sap as carbon sources (i.e., sugars) to fulfill proliferation in xylem vessels (Lowe-Power et al. 2018a and b). Plants synthesize abundant secondary metabolites and bioactive compounds in xylem vessels, and nutrients in plants are relatively more abundant than in the sucrose medium (minimal medium). It is not striking that the $\mathrm{RSc} 0454$ mutant fails to grow in the minimal medium but can grow faintly in host plants. The condition in xylem vessels is known to be low in oxygen, and $R$. solanacearum uses nitrate, which is rich in xylem sap, as an alternate electron acceptor for cell respiration to fulfill proliferation in the hypoxic xylem environment (Dalsing et al. 2015; Jacobs et al. 2012). In addition to $\mathrm{RSc0454,} R$. solanacearum has authentic membrane-bound SDHs (i.e., Rsp1195 and Rsp1196) encoding a putative transmembrane SDH anchor subunit and cytochrome B-556 subunit, respectively, and Rsc0028 and Rsp1611 encoding putative succinate-semialdehyde dehydrogenases. Therefore, the $\mathrm{RSc0454}$

Table 1. Bacterial strains used in this study

\begin{tabular}{|c|c|c|}
\hline Strain & Relative characteristics ${ }^{a}$ & References \\
\hline \multicolumn{3}{|l|}{ Ralstonia solanacearum } \\
\hline OE1-1 & Wild-type, race 1 , biovar 3 & Kanda et al. 2003 \\
\hline RK5043 & OE1-1, phcA-lacZYA & Yoshimochi et al. 2009b \\
\hline RK5046 & OE1-1, hrpB-lacZYA & Yoshimochi et al. 2009b \\
\hline RK5050 & OE1-1, popA-lacZYA & Yoshimochi et al. 2009b \\
\hline RK5120 & OE1-1, hrpG-lacZYA & Yoshimochi et al. 2009b \\
\hline RK5124 & OE1-1, prhJ-lacZYA & Yoshimochi et al. 2009b \\
\hline RK5212 & OE1-1, prhG-lacZYA & Zhang et al. 2013 \\
\hline RK5619 & OE1-1, prhN-lacZYA & Zhang et al. 2015 \\
\hline RQ5699 & RK5050, $\Delta r s c 0454$ & This study \\
\hline RQ5932 & RK5046, $\Delta r s c 0454$ & This study \\
\hline RQ5987 & RK5120, $\Delta r s c 0454$ & This study \\
\hline RQ5990 & RK5212, $\Delta r s c 0454$ & This study \\
\hline RQ6081 & RK5124, $\Delta r s c 0454$ & This study \\
\hline RQ6081 & RK5619, $\Delta r s c 0454$ & This study \\
\hline RQ6115 & RK5043, $\Delta r s c 0454$ & This study \\
\hline RQC0307 & Complementary rsc0454 in RQ5699 & This study \\
\hline \multicolumn{3}{|l|}{ Escherichia coli } \\
\hline DH12S & Host strain for standard DNA cloning manipulation & Invitrogen \\
\hline S17-1 & Host strain for plasmid mobilization & Simon et al. 1983 \\
\hline \multicolumn{3}{|c|}{ (1) } \\
\hline pK18mobsacB & $s a c B$, lacZa, $\mathrm{Km}^{\mathrm{r}}$; cloning vector for allelic exchange & Simon et al. 1983 \\
\hline pUC18-miniTn7T-Gm & $\mathrm{Gm}^{\mathrm{r}}$ on mini-Tn7T; mobilizable & Choi et al. 2005 \\
\hline pTNS2 & $\begin{array}{l}\mathrm{Ap}^{\mathrm{r}} \text {; helper plasmid encoding the site-specific TnsABCD } \\
\text { Tn7 transposition pathway }\end{array}$ & Choi et al. 2005 \\
\hline
\end{tabular}

\footnotetext{
${ }^{\mathrm{a}} \mathrm{Km}^{\mathrm{r}}, \mathrm{Gm}^{\mathrm{r}}$, and $\mathrm{Ap}^{\mathrm{r}}$ and indicate resistant to kanamycin, gentamicin, and ampicillin, respectively.
} 
mutant is not a simple auxotroph. It was consistent with previous reports that, in addition to catalytic activities for redox reaction directly, $\mathrm{LDH}$ and SDH are essential for cell proliferation, membrane generation, and virulence in many microbes (Abdelbaset et al. 2017; Kim et al. 2017; Mercado-Lubo et al. 2008; Pecsi et al. 2014).

RSc0454 was previously screened as one of the T3SSaffecting candidates (Zhang et al. 2013). Here, we experimentally confirmed that $\mathrm{RSc0454}$ is required for expression of the T3SS both in vitro and in planta. The T3SS expression of the RSc0454 mutant in the minimal medium was substantially decreased even in the presence of supplementary succinate at 40 $\mathrm{mM}$ that partially restored diminished growth of the $\mathrm{RSc} 0454$ mutant in the minimal medium, confirming that impaired expression of the T3SS in the RSc0454 mutant was not due to growth deficiency in the minimal medium. Moreover, supplementary succinate did not restore expression of the T3SS in the RSc0454 mutant inside host plants, confirming that involvement of RSc0454 in T3SS expression is possibly due to the unbalanced redox reaction inside cells but not growth deficiency under nutrient-limited conditions. The $h r p B$ expression was significantly impaired in the $\mathrm{RSc} 0454$ mutant. It was consistent with the master role of $\mathrm{HrpB}$ that directly controls expression of the entire T3SS and T3Es genes (Mukaihara et al. 2004; Valls et al. 2006). Two close paralogues (HrpG and PrhG) positively regulate $h r p B$ expression in parallel ways (Plener et al. 2010; Zhang et al. 2013). Deletion of $R S c 0454$ substantially impaired expression of both $h r p G$ and $p r h G$, whereas deletion of $R S c 0454$ did not alter expression of prhJ, phcA, and prhN, which positively regulate expression of $h r p G$ or $p r h G$, respectively, indicating that the RSc0454-based redox balance is possibly important for expression of $h r p G$ and $p r h G$ through some novel pathway. It is not a special case and we previously reported that a plant-derived compound, umbelliferone, impairs the T3SS via pathway of HrpG and PrhG to HrpB but is independent of known pathways (Yang et al. 2017). A novel regulator (PrhO) positively regulates expression of $h r p G$ and $p r h G$ to HrpB through some novel pathway (Zhang et al. 2018), supporting our speculation that some novel pathways should be integrated for expression of $h r p G$ and $p r h G$ in $R$. solanacearum.

Taken together, these results provide novel insight into understanding the biological functions of this FAD-linked oxidase and requirement of the $\mathrm{RSc0454-based} \mathrm{redox} \mathrm{balance} \mathrm{for} \mathrm{expression}$ of the T3SS in $R$. solanacearum.

\section{AUTHOR-RECOMMENDED INTERNET RESOURCE}

Ralstonia solanacearum database:

https://iant.toulouse.inra.fr/bacteria/annotation/cgi/ralso.cgi

\section{LITERATURE CITED}

Abdelbaset, A. E., Fox, B. A., Karram, M. H., Abd Ellah, M. R., Bzik, D. J., and Igarashi, M. 2017. Lactate dehydrogenase in Toxoplasma gondii controls virulence, bradyzoite differentiation, and chronic infection. PLoS One 12:e0173745.

Aldon, D., Brito, B., Boucher, C., and Genin, S. 2000. A bacterial sensor of plant cell contact controls the transcriptional induction of Ralstonia solanacearum pathogenicity genes. EMBO J. 19:2304-2314.

Angot, A., Peeters, N., Lechner, E., Vailleau, F., Baud, C., Gentzbittel, L., Sartorel, E., Genschik, P., Boucher, C., and Genin, S. 2006. Ralstonia solanacearum requires F-box-like domain-containing type III effectors to promote disease on several host plants. Proc. Natl. Acad. Sci. U.S.A. 103:14620-14625

Arlat, M., Gough, C. L., Zischek, C., Barberis, P. A., Trigalet, A., and Boucher, C. A. 1992. Transcriptional organization and expression of the large hrp gene cluster of Pseudomonas solanacearum. Mol. PlantMicrobe Interact. 5:187-193.
Choi, K. H., Gaynor, J. B., White, K. G., Lopez, C., Bosio, C. M., Karkhoff-Schweizer, R. R., and Schweizer, H. P. 2005. A Tn7-based broad-range bacterial cloning and expression system. Nat. Methods 2: 443-448.

Coll, N. S., and Valls, M. 2013. Current knowledge on the Ralstonia solanacearum type III secretion system. Microb. Biotechnol. 6:614-620.

Cunnac, S., Occhialini, A., Barberis, P., Boucher, C., and Genin, S. 2004. Inventory and functional analysis of the large Hrp regulon in Ralstonia solanacearum: Identification of novel effector proteins translocated to plant host cells through the type III secretion system. Mol. Microbiol. 53:115-128.

Dalsing, B. L., Truchon, A. N., Gonzalez-Orta, E. T., Milling, A. S., and Allen, C. 2015. Ralstonia solanacearum uses inorganic nitrogen metabolism for virulence, ATP production, and detoxification in the oxygen-limited host xylem environment. MBio 6:e02471.

Denny, T. P. 1995. Involvement of bacterial polysaccharides in plant pathogenesis. Annu. Rev. Phytopathol. 33:173-197.

Evert, R. F., and Eichhorn, S. E. 2006. Esau's Plant Anatomy: Meristems, Cells, and Tissues of the Plant Body: Their Structure, Function, and Development, Third Edition. John Wiley \& Sons, Inc., Hoboken, NJ, U.S.A.

Gao, C., Wang, Y., Zhang, Y., Lv, M., Dou, P., Xu, P., and Ma, C. 2015. NAD-independent L-lactate dehydrogenase required for L-lactate utilization in Pseudomonas stutzeri A1501. J. Bacteriol. 197:2239-2247.

Genin, S. 2010. Molecular traits controlling host range and adaptation to plants in Ralstonia solanacearum. New Phytol. 187:920-928.

Genin, S., Brito, B., Denny, T. P., and Boucher, C. 2005. Control of the Ralstonia solanacearum Type III secretion system (Hrp) genes by the global virulence regulator PhcA. FEBS Lett. 579:2077-2081.

Genin, S., and Denny, T. P. 2012. Pathogenomics of the Ralstonia solanacearum species complex. Annu. Rev. Phytopathol. 50:67-89.

Heikal, A., Nakatani, Y., Dunn, E., Weimar, M. R., Day, C. L., Baker, E. N., Lott, J. S., Sazanov, L. A., and Cook, G. M. 2014. Structure of the bacterial type II NADH dehydrogenase: A monotopic membrane protein with an essential role in energy generation. Mol. Microbiol. 91:950-964.

Hikichi, Y., Mori, Y., Ishikawa, S., Hayashi, K., Ohnishi, K., Kiba, A., and Kai, K. 2017. Regulation involved in colonization of intercellular spaces of host plants in Ralstonia solanacearum. Front. Plant Sci. 8:967.

Hu, X., Zhao, Z., Zhuo, T., Fan, X., and Zou, H. 2019. The RSc0454encoded FAD-linked oxidase is indispensable for pathogenicity in Ralstonia solanacearum GMI1000. Mol. Plant-Microbe Interact. 32: 697-707.

Huynen, M. A., Dandekar, T., and Bork, P. 1999. Variation and evolution of the citric-acid cycle: A genomic perspective. Trends Microbiol. 7: 281-291.

Jacobs, J. M., Babujee, L., Meng, F., Milling, A., and Allen, C. 2012. The in planta transcriptome of Ralstonia solanacearum: Conserved physiological and virulence strategies during bacterial wilt of tomato. MBio 3:e00114-12.

Jones, J. D. G., and Dangl, J. L. 2006. The plant immune system. Nature 444:323-329.

Kanda, A., Ohnishi, S., Tomiyama, H., Hasegawa, H., Yasukohchi, M., Kiba, A., Ohnishi, K., Okuno, T., and Hikichi, Y. 2003. Type III secretion machinery-deficient mutants of Ralstonia solanacearum lose their ability to colonize resulting in loss of pathogenicity. J. Gen. Plant Pathol. 69:250-257.

Kim, J. H., Bae, K. H., Byun, J. K., Lee, S., Kim, J. G., Lee, I. K., Jung, G. S., Lee, Y. M., and Park, K. G. 2017. Lactate dehydrogenase-A is indispensable for vascular smooth muscle cell proliferation and migration. Biochem. Biophys. Res. Commun. 492:41-47.

Lowe-Power, T. M., Hendrich, C. G., von Roepenack-Lahaye, E., Li, B., Wu, D., Mitra, R., Dalsing, B. L., Ricca, P., Naidoo, J., Cook, D. Jancewicz, A., Masson, P., Thomma, B., Lahaye, T., Michael, A. J., and Allen, C. 2018a. Metabolomics of tomato xylem sap during bacterial wilt reveals Ralstonia solanacearum produces abundant putrescine, a metabolite that accelerates wilt disease. Environ. Microbiol. 20: 1330-1349.

Lowe-Power, T. M., Khokhani, D., and Allen, C. 2018b. How Ralstonia solanacearum exploits and thrives in the flowing plant xylem environment. Trends Microbiol. 26:929-942.

Mansfield, J., Genin, S., Magori, S., Citovsky, V., Sriariyanum, M., Ronald, P., Dow, M., Verdier, V., Beer, S. V., Machado, M. A., Toth, I., Salmond, G., and Foster, G. D. 2012. Top 10 plant pathogenic bacteria in molecular plant pathology. Mol. Plant Pathol. 13:614-629.

Marenda, M., Brito, B., Callard, D., Genin, S., Barberis, P., Boucher, C., and Arlat, M. 1998. PrhA controls a novel regulatory pathway required 
for the specific induction of Ralstonia solanacearum hrp genes in the presence of plant cells. Mol. Microbiol. 27:437-453.

Mercado-Lubo, R., Gauger, E. J., Leatham, M. P., Conway, T., and Cohen, P. S. 2008. A Salmonella enterica serovar typhimurium succinate dehydrogenase/fumarate reductase double mutant is avirulent and immunogenic in BALB/c mice. Infect. Immun. 76:1128-1134.

Mukaihara, T., Tamura, N., and Iwabuchi, M. 2010. Genome-wide identification of a large repertoire of Ralstonia solanacearum type III effector proteins by a new functional screen. Mol. Plant-Microbe Interact. 23:251-262.

Mukaihara, T., Tamura, N., Murata, Y., and Iwabuchi, M. 2004. Genetic screening of Hrp type III-related pathogenicity genes controlled by the HrpB transcriptional activator in Ralstonia solanacearum. Mol. Microbiol. 54:863-875.

Park, D. H., Laivenieks, M., Guettler, M. V., Jain, M. K., and Zeikus, J. G. 1999. Microbial utilization of electrically reduced neutral red as the sole electron donor for growth and metabolite production. Appl. Environ. Microbiol. 65:2912-2917.

Pecsi, I., Hards, K., Ekanayaka, N., Berney, M., Hartman, T., Jacobs, W. R., Jr., and Cook, G. M. 2014. Essentiality of succinate dehydrogenase in Mycobacterium smegmatis and its role in the generation of the membrane potential under hypoxia. MBio 5:e01093-14.

Pegg, G. F. 1985. Life in a black hole-The micro-environment of the vascular pathogen. Trans. Br. Mycol. Soc. 85:IN1, 1-20.

Plener, L., Manfredi, P., Valls, M., and Genin, S. 2010. PrhG, a transcriptional regulator responding to growth conditions, is involved in the control of the type III secretion system regulon in Ralstonia solanacearum. J. Bacteriol. 192:1011-1019.

Poueymiro, M., Cunnac, S., Barberis, P., Deslandes, L., Peeters, N., Cazale-Noel, A. C., Boucher, C., and Genin, S. 2009. Two type III secretion system effectors from Ralstonia solanacearum GMI1000 determine host-range specificity on tobacco. Mol. Plant-Microbe Interact. 22:538-550.

Poueymiro, M., and Genin, S. 2009. Secreted proteins from Ralstonia solanacearum: A hundred tricks to kill a plant. Curr. Opin. Microbiol. 12:44-52.

Roberts, D. P., Denny, T. P., and Schell, M. A. 1988. Cloning of the egl gene of Pseudomonas solanacearum and analysis of its role in phytopathogenicity. J. Bacteriol. 170:1445-1451.

Salanoubat, M., Genin, S., Artiguenave, F., Gouzy, J., Mangenot, S., Arlat M., Billault, A., Brottier, P., Camus, J. C., Cattolico, L., Chandler, M., Choisne, N., Claudel-Renard, C., Cunnac, S., Demange, N., Gaspin, C., Lavie, M., Moisan, A., Robert, C., Saurin, W., Schiex, T., Siguier, P., Thébault, P., Whalen, M., Wincker, P., Levy, M., Weissenbach, J., and
Boucher, C. A. 2002. Genome sequence of the plant pathogen Ralstonia solanacearum. Nature 415:497-502.

Simon, R., Priefer, U., and Pühler, A. 1983. A broad host range mobilization system for in vivo genetic engineering: Transposon mutagenesis in gram negative bacteria. Nat. Biotechnol 1:784-791.

Valls, M., Genin, S., and Boucher, C. 2006. Integrated regulation of the type III secretion system and other virulence determinants in Ralstonia solanacearum. PLoS Pathog. 2:e82.

Vasse, J., Frey, P., and Trigalet, A. 1995. Microscopic studies of intercellular infection and protoxylem invasion of tomato roots by Pseudomonas solanacearum. Mol. Plant-Microbe Interact. 8:241-251.

Yang, L., Li, S., Qin, X., Jiang, G., Chen, J., Li, B., Yao, X., Liang, P. Zhang, Y., and Ding, W. 2017. Exposure to umbelliferone reduces Ralstonia solanacearum biofilm formation, transcription of type III secretion system regulators and effectors and virulence on tobacco. Front. Microbiol. 8:1234.

Yoshimochi, T., Hikichi, Y., Kiba, A., and Ohnishi, K. 2009a. The global virulence regulator PhcA negatively controls the Ralstonia solanacearum hrp regulatory cascade by repressing expression of the PrhIR signaling proteins. J. Bacteriol. 191:3424-3428.

Yoshimochi, T., Zhang, Y., Kiba, A., Hikichi, Y., and Ohnishi, K. 2009b. Expression of $h r p G$ and activation of response regulator HrpG are controlled by distinct signal cascades in Ralstonia solanacearum. J. Gen. Plant Pathol. 75:196-204.

Zhang, Y., Cao, Y., Zhang, L., Ohnishi, K., Hikichi, Y., and Li, J. 2021. The Tn7-based genomic integration is dependent on an att $\operatorname{Tn} 7$ box in the glms gene and is site-specific with monocopy in Ralstonia solanacearum species complex. Mol. Plant-Microbe Interact. 34:720-725.

Zhang, Y., Chen, L., Yoshimochi, T., Kiba, A., Hikichi, Y., and Ohnishi, K. 2013. Functional analysis of Ralstonia solanacearum PrhG regulating the hrp regulon in host plants. Microbiol. Read. 159:1695-1704.

Zhang, Y., Kiba, A., Hikichi, Y., and Ohnishi, K. 2011. prhKLM genes of Ralstonia solanacearum encode novel activators of hrp regulon and are required for pathogenesis in tomato. FEMS Microbiol. Lett. 317: 75-82.

Zhang, Y., Li, J., Zhang, W., Shi, H., Luo, F., Hikichi, Y., Shi, X., and Ohnishi, K. 2018. A putative LysR-type transcriptional regulator PrhO positively regulates the type III secretion system and contributes to the virulence of Ralstonia solanacearum. Mol. Plant Pathol. 19:1808-1819.

Zhang, Y., Luo, F., Wu, D., Hikichi, Y., Kiba, A., Igarashi, Y., Ding, W., and Ohnishi, K. 2015. PrhN, a putative marR family transcriptional regulator, is involved in positive regulation of type III secretion system and full virulence of Ralstonia solanacearum. Front. Microbiol. 6:357. 